\title{
CORRESPONDENCE
}

\section{India's lockdown exit: are we prepared to lock horns with COVID-19 and dengue in the rainy season?}

Pediatric Research (2021) 89:1047-1048; https://doi.org/10.1038/s41390020-1063-7

Novel coronavirus (SARS-CoV-2) infection causing coronavirus disease (COVID-19) was first reported in India in late January 2020 among students who returned from Wuhan, China. The disease transmission escalated in March, reaching 100 cases by mid-March and crossing 1000 cases by the end of the month. The government enforced world's largest nationwide lockdown on March 24, 2020 for 21 days, further extending it twice for 2 weeks each. This tough and timely measure, as quoted by WHO, has given the country's strained health system time to prepare for the forecasted surge in number of infections. ${ }^{1}$ At the time of publication, 719,665 infections were confirmed with a steady increase in daily reported cases. ${ }^{2}$ The lockdown has quite possibly delayed the peak of the pandemic in India, postponing it to the end of summer (July-September), the months that mark the onset of monsoon in several states.

The rainy and post rainy periods between July and October in India report the bulk of seasonal infections such as dengue, scrub typhus, malaria and other tropical fevers. Dengue is widely prevalent among all ages and known to present with short history of undifferentiated fever. ${ }^{3}$ When both dengue and COVID-19 reach epidemic proportions at the same time, it may be difficult to distinguish these two illnesses particularly in outpatient settings. The initial clinical symptoms such as fever, headache and myalgia are common to both illnesses, though the presence of respiratory symptoms such as cough, sore throat and new loss of taste or smell may favour the diagnosis of COVID-19. The implications of potential temporal overlap of these two outbreaks can be fourfold. Firstly, the laboratory diagnosis of individual infections would become a challenging task. Being a systemic viral infection, diagnosis of dengue largely relies on serological tests. ELISA-based methods are recommended; nevertheless, point-of-care card tests are increasingly being used due to ease of performance and rapidity of results. Recent report from Singapore of false-positive card tests for dengue in two patients with SARS-CoV-2 infection raises serious concern in the reliability of these commercially available tests during COVID-19 pandemic. ${ }^{4}$ Secondly, the effect of possible coinfection with dengue and SARS-CoV-2 or the clinical course of dengue in a recently recovered COVID-19 patient is unknown. Much remains to be understood about the immunopathogenesis of severe disease in dengue. Extent of transient autoimmunity with anti NS-1 antibodies, phenomenon of pre-immunity and antibodydependent enhancement and role of cross reactive T-cell response in the development of pathological changes during secondary infection with dengue virus need to be carefully studied in the context of recent or concurrent SARS-CoV-2induced immunological changes. ${ }^{5,6}$ Thirdly, emerging reports of hyperinflammatory syndrome associated with COVID-19 in children mimicking toxic shock and Kawasaki disease further complicate the interpretation in the face of coinfections. ${ }^{7}$

Lastly, the acute public health emergency when these two illnesses peak in tandem would be a significant challenge to handle. A cross-sectional population-based serosurvey in India estimated 8.8-12.9 million primary dengue infections in 2017 among individuals aged $5-45$ years. Assuming about $25 \%$ to be symptomatic infection, the burden of clinical cases would be 2.2-3.2 million. ${ }^{8}$ These figures draw parallel to COVID-19 trend projections for India, predicting a million cases in few months, most asymptomatic but the symptomatic and severe ones competing for limited hospital beds. ${ }^{9}$ The burden of both diseases being borne by crowded urban areas could make the matters worse. While we exit from the lockdown, we should have a multi-pronged approach to early case detection, isolation and management of not only COVID-19 case, but also various febrile illnesses that visit us during these premonsoon and monsoon times. As physical and social distancing becomes a norm in this changed world, so should vector control and effective management of urban and household waters.

\section{AUTHOR CONTRIBUTIONS}

N.D. and K.N. conceptualized the paper. N.D. and K.N. wrote the first draft. W.R. provided critical inputs and contributed to the final version of the manuscript. All authors read and approved the final manuscript.

\section{ADDITIONAL INFORMATION}

Competing interests: The authors declare no competing interests.

Publisher's note Springer Nature remains neutral with regard to jurisdictional claims in published maps and institutional affiliations.

Nabaneeta Dash ${ }^{1}$, Winsley Rose $^{1}$ and Karthi Nallasamy ${ }^{2}$

${ }^{1}$ Pediatric Infectious Disease Unit, Department of Pediatrics, Christian Medical College, Vellore, India and ${ }^{2}$ Pediatric Intensive Care Unit, Department of Pediatrics, Postgraduate Institute of Medical Education and Research, Chandigarh, India Correspondence: Karthi Nallasamy (ny.karthi@gmail.com)

\section{REFERENCES}

1. The Lancet. India under COVID-19 lockdown. Lancet 395, 1315 (2020).

2. World Health Organization. Situation report-108. Coronavirus disease 2019 (COVID-19), 8 May 2020. https://www.who.int/docs/default-source/coronaviruse/ situation-reports/20200508covid-19-sitrep-109.pdf?sfvrsn=68f2c632_6 (2020).

3. Singhi, S. et al. Tropical fevers in Indian intensive care units: a prospective multicenter study. Indian J. Crit. Care Med. 21, 811-818 (2017).

4. Yan, G. et al. Covert COVID-19 and false-positive dengue serology in Singapore. Lancet Infect. Dis. 20, 536 (2020).

5. Martina, B. E., Koraka, P. \& Osterhaus, A. D. Dengue virus pathogenesis: an integrated view. Clin. Microbiol. Rev. 22, 564-581 (2009). 
1048

6. Pang, X., Zhang, R. \& Cheng, G. Progress towards understanding the pathogenesis of dengue hemorrhagic fever. Virol. Sin. 32, 16-22 (2017).

7. Riphagen, S., Gomez, X., Gonzalez-Martinez, C., Wilkinson, N. \& Theocharis, P. Hyperinflammatory shock in children during COVID-19 pandemic. Lancet https:// doi.org/10.1016/S0140-6736(20)31094-1 (2020).
8. Murhekar, M. V. et al. Burden of dengue infection in India, 2017: a crosssectional population based serosurvey. Lancet Glob. Health 7, e1065-e1073 2019)

9. Tiwari, S., Kumar, S. \& Guleria, K. Outbreak trends of CoronaVirus (COVID-19) in India: a prediction. Disaster Med. Public Health Prep. 22, 1-9 (2020). 